Ia

Révolution

française

\section{La Révolution française}

Cahiers de l'Institut d'histoire de la Révolution française

$17 \mid 2020$

Gouverner par la loi

\title{
De la poétique de la foule à la politique de la foule dans 14 juillet d'Éric Vuillard
}

Louis Autin, Pierre-Victor Haurens et Yves Schulze

\section{(2) OpenEdition}

\section{Journals}

Édition électronique

URL : http://journals.openedition.org/lrf/3532

DOI : $10.4000 /$ Irf.3532

ISSN : 2105-2557

Éditeur

IHMC - Institut d'histoire moderne et contemporaine (UMR 8066)

Référence électronique

Louis Autin, Pierre-Victor Haurens et Yves Schulze, « De la poétique de la foule à la politique de la foule dans 74 juillet d'Éric Vuillard», La Révolution française [En ligne], 17| 2020, mis en ligne le 24 février 2020, consulté le 20 avril 2020. URL : http://journals.openedition.org//rf/3532 ; DOI : https://doi.org/ $10.4000 /$ Irf.3532

Ce document a été généré automatiquement le 20 avril 2020.

(c) La Révolution française 


\title{
De la poétique de la foule à la politique de la foule dans 14 juillet d'Éric Vuillard
}

\author{
Louis Autin, Pierre-Victor Haurens et Yves Schulze
}

\section{NOTE DE L'AUTEUR}

Cet article est né d'un atelier d'écriture organisé dans le cadre des formations de l'ED 3LA de Lyon par Marie-Jeanne Zenetti, en collaboration avec le festival des Assises Internationales du Roman (AIR) 2017. Nous tenons donc à remercier chaleureusement Marie-Jeanne Zenetti, dont les relectures et les conseils divers ont grandement contribué à améliorer ce travail.

\section{Introduction}

1 "Qu'est-ce que c'est, une foule ? Personne ne veut le dire ${ }^{1}$ ", déclare Éric Vuillard dans 14 juillet, « récit » (pour reprendre l'appellation générique donnée sur la page de titre) publié en 2016 et faisant partie des œuvres à sujet historique de l'auteur. En première lecture, cette déclaration semble rendre difficile l'étude que nous abordons de la représentation de la foule révolutionnaire dans ce texte, d'autant plus qu'É. Vuillard n'entend pas tout à fait aller à l'encontre de ce refus. De fait, nous ne trouverons pas ici de définition d'essence, de ti esti grec; en effet, celui-ci est, par nature, un discours de maitrise, et ultimement, un discours sur, tandis que la perspective d'éric Vuillard dans 14 juillet est, elle, différente : le récit sera écrit depuis la foule ${ }^{2}$. L'œuvre a pour but de «raconter » et non d'" enseigner » (p.33), et il s'agit d'abord de combler un vide narratif, l'absence des petites gens de l'historiographie traditionnelle de la Révolution française ${ }^{3}$. À ce titre, les récits d'É. Vuillard s'inscrivent, comme cela a déjà été dit à de 
nombreuses reprises, dans un rapport à la fois proche et polémique à l'Histoire, et à l'écriture de l'histoire comme discipline ${ }^{4}$.

2 Nous chercherons ici moins à suivre cette piste frontalement qu'à prendre au sérieux l'idée selon laquelle le style et les partis pris qu'il implique constituent une forme-sens, qui permet de s'approcher au plus près de la réalité, ici celle de la foule, à défaut de pouvoir en dire exactement la vérité ou le secret : par sa plasticité, la littérature serait plus à même de parler de la masse agissante que l'historiographie, aussi bien dans ses atours littéraires $\mathrm{du}$ XIX $\mathrm{X}^{\mathrm{e}}$ siècle que dans ses réalités académiques contemporaines. À partir de là, nous chercherons à voir et à montrer comment l'écriture de la foule dans 14 juillet, sa poétique, contient toujours, d'ores et déjà, une politique de la foule. Pour ce faire, nous partons de certains phénomènes stylistiques marquants, au niveau de la phrase ou du paragraphe, comme la liste et l'emploi du pronom indéfini on, avant d'élargir notre analyse à la composition du récit et à l'image de la foule qui se constitue à travers la représentation des discours qui portent sur elle. On verra ainsi comment le texte, dans toute sa littérarité, se constitue comme un creuset où se mêlent plusieurs éléments : une lecture personnelle des événements de juillet 1789, une contestation fortement polémique de l'historiographie - notamment républicaine - de la Révolution française, une certaine influence de la philosophie politique et de la pensée de l'insurrection (notamment $\mathrm{J}$. Rancière) et, enfin, une profonde sensibilité à la matérialité textuelle de la foule.

3 Pour mener cette enquête, nous varierons donc les échelles, passant de la phrase au récit, et du récit au discours. On ne saurait oublier un préalable indispensable : celui du mot, de l'unité linguistique minimale qui cimente chacun de ces niveaux. Une brève analyse nous évitera toute confusion lexicale dans la suite de cet article. Qu'est-ce qu'une foule pour É. Vuillard? Sans aller jusqu'à affirmer que l'auteur développe un système sémantique parfaitement cohérent et pensé comme tel, force est de constater que des concepts assez bien délimités s'articulent chez lui à un vocabulaire précis, qui permet bien de reconstituer une sorte de phénoménologie du soulèvement. Cela commence avec des gens, peu intéressés ou peu mobilisés ${ }^{5}$; viennent ensuite les groupes, encore trop disjoints pour prétendre peser sur l'Histoire ${ }^{6}$. Plus structurée, la masse a, quant à elle, le bénéfice du regroupement et du nombre ; elle est pourtant passive et ne paraît pas viser à l'action, à la différence de la foule, qui occupe le cœur du récit ${ }^{7}$. La phénoménologie de l'agir collectif que traduisent, toujours en filigrane, les choix de vocabulaire de l'auteur ne sont certes pas le résultat d'une étude scientifique du phénomène révolutionnaire, qui irait de toute façon à l'encontre du refus définitoire de l'auteur; il n'en reste pas moins que la typologie d'un historien comme Georges Lefebvre n'en est, en dernière instance, pas très éloignée ${ }^{8}$. Reste une dernière distinction, fondamentale : celle qui s'établit entre la foule et le peuple. Les deux termes semblent souvent synonymes, comme l'attestent les effets de reformulation et les tâtonnements du récit : « la petite forêt des témoignages [...] s'efface à mesure que l'on va vers la foule, à mesure qu'on s'approche du peuple » (p. 92). En réalité, ils ne se situent pas sur le même plan. La foule se définit dans son existence ponctuelle, dans son rapport étroit à l'action; le peuple se trouve, lui, en aval de cette réalité : son essence est politique. Dans la perspective d'un politicien comme Camille Desmoulins, l'orateur s'adresse au peuple ; mais quand on évoque les motivations des émeutiers, quelques lignes plus loin, c'est bien de la foule dont il s'agit ${ }^{9}$. 
« Hier vous n'étiez qu'une foule. / Vous êtes un peuple aujourd'hui !», s'exclamait Hugo dans des vers bien connus («Dicté après juillet 1830 », IV, Les Chants du crépuscule, 1835). Si É. Vuillard reprend à son compte cette dichotomie, il rejette la vision négative de la foule qui s'y attache. Ainsi la perspective se renverse-t-elle dans 14 juillet : l'auteur cherche à rendre sa dignité à l'aujourd'hui de la foule, et laisse au peuple les promesses du demain. Ce sont les mécanismes stylistiques, génériques et discursifs d'un tel choix qu'il s'agit à présent d'analyser.

\section{Le stylistique dans 14 juillet : une écriture politique de la foule}

5 Si l'on accepte de considérer l'ouvrage d'É. Vuillard comme une réflexion en partie historique sur la Révolution française, son apport décisif à l'historiographie de la prise de la Bastille ne réside pas en propre dans sa focalisation sur les foules comme agents décisifs de l'événement révolutionnaire, ni dans sa réévaluation de leurs motivations ou de leur comportement. Dès 1959, l'historien marxiste Georges Rudé, proche de l'École des Annales et de Georges Lefebvre, s'intéressait à la composition des groupes d'émeutiers de la Révolution et prouvait, en se fondant sur des sources alors peu mobilisées, à l'instar des procès-verbaux de police, qu'il s'agissait surtout de la partie médiane de la population parisienne (artisans, boutiquiers) ${ }^{10}$, dont les motivations étaient surtout économiques ${ }^{11}$. L'objectif et la méthode (notamment les sources) sont similaires à ceux de 14 juillet. La proposition originale d'É. Vuillard est bien plutôt celle d'une mise en adéquation entre un regard non discréditant sur les foules et une poétique particulière, tranchant avec les modèles narratifs traditionnels et l'idéologie qui s'y attache ; elle vise donc à synchroniser la forme et le sens, la célébration politique des foules agissantes et leur représentation littéraire. En ce sens, elle ne peut se comprendre qu'en rupture avec une tradition dont il s'agit de rappeler ici brièvement les grandes lignes. Ayant effectué ce rappel et situé É. Vuillard dans un continuum historiographique, nous évoquerons les deux traits stylistiques les plus saillants, à notre sens, de cette poétique novatrice des foules, qui ne pouvait prendre forme qu'en empruntant au roman, sinon au romanesque, certaines de ses ressources : l'utilisation de la liste comme structure totale, et le jeu sur l'indéfini, en particulier sur le pronom on.

\section{Contre-modèles : essentialisation et individualisation des foules}

6 La fréquence des références d'É. Vuillard à ses modèles littéraires et politiques autorise qu'on pense son œuvre en relation avec les auteurs qui, avant lui, se sont intéressés aux foules et à leur représentation; aussi évoquerons-nous en quelques mots la longue tradition contre laquelle l'auteur de 14 juillet semble s'inscrire en faux. Celle-ci remonte en dernière instance à l'Antiquité. Il est à noter qu'on ne trouve pas chez les Anciens de réflexion sur la poétique des foules à proprement parler : la question, de fait, ne prête pas à débat, dans une vision de l'Histoire où les individus issus de l'élite agissent, et où la masse suit. Le fait littéraire dans l'Antiquité, tout accaparé qu'il est par une élite hégémonique aux plans politique, culturel et social, ne peut structurellement aboutir qu'à une représentation de la foule biaisée, que nous nommerons ici unitaire: celle-ci est en quelque sorte vue de dessus, dans une perspective qui ne cherche pas à 
distinguer des structurations internes, et uniformisée dans son action ${ }^{12}$. Cette poétique massive résiste aux variations axiologiques, faibles en réalité, qui se manifestent d'un auteur à un autre quand il s'agit de classes subalternes. Ainsi, dans la philosophie politique athénienne, si Aristote a beau jeu de célébrer la poikilia (mélange, diversité) qui constitue la masse et l'élève ${ }^{13}$, notamment face au mépris de Platon pour le " gros animal » qu'est le dèmos ${ }^{14}$, l'un comme l'autre la traitent non comme une somme d'acteurs individualisés, mais comme un personnage collectif, à la psychologie unique et aux sentiments communs. Cette conception des foules traverse les ères et les genres : elle rapproche l'historiographie d'un Thucydide ou d'un Tacite, l'épopée primitive d'Homère et celle d'époque flavienne, ou encore la tragédie grecque qui, selon les thèses bien connues de J.-P. Vernant, intègre le peuple sous la forme d'un personnage collectif, le chœur et singulièrement le coryphée ${ }^{15}$. L'historiographie du $\mathrm{xIx}^{\mathrm{e}}$ siècle, malgré les courants idéologiques divergents qui la parcourent, n'a pas réellement conduit de réflexion quant aux formes particulières que pourrait prendre une narration des foules : témoin l'égalité structurelle entre l'exaltation chez Michelet du "peuple " en marche et le dégoût de Taine pour "la dernière plèbe " ${ }^{16}$. Le point d'aboutissement de ce mouvement semble bien être, pour son pôle négatif en tout cas, la tentative de Le Bon de fonder une psychologie sociale moderne des foules, tout empreinte de peur, d'incompréhension et de mépris pour ces groupes humains, sur une méconnaissance complète de ses réseaux et de sa structuration internes ${ }^{17}$.

$7 \mathrm{Au}$ XIX ${ }^{\mathrm{e}}$ siècle, c'est dans le genre romanesque que l'effort pour prendre en compte l'entrée du peuple sur la scène de l'Histoire (pour reprendre une expression courante, mais utilisée par É. Vuillard ${ }^{18}$ ) produit les résultats les plus visibles: on pense notamment au motif du jeune ambitieux, de basse extraction, sur le parcours duquel le romancier se concentre. Rejoignant en cela par certains côtés le modèle picaresque ${ }^{19}$, la tentation romantique et réaliste, par sa focalisation exclusive sur l'individu, délaisse la question de la représentation spécifique de la masse, et ne constitue pas un modèle pour l'auteur de 14 juillet, qui rappelle explicitement la préexistence de la foule révolutionnaire à ces héros romanesques ${ }^{20}$. Au reste, É. Vuillard semble également rejeter la poursuite de ce modèle à l'époque contemporaine dans la micro-histoire de Carlo Ginzburg (pour son volet scientifique) et dans les Vies minuscules de Pierre Michon (pour son volet fictionnel ou autofictionnel): la tentation d'écrire des destins minuscules affleure parfois ${ }^{21}$, mais se limite toujours à des évocations parcellaires et distantes. Le refus, ou l'impossibilité, de constituer une psychologie propre aux personnages ou même d'instituer des héros est patent, en particulier dans la présence de « personnages disparaissants ${ }^{22}$ », qui ne livrent d'eux que des éclats de portrait.

8 Dès lors et pour résumer, l'approche de É. Vuillard n'est pas d'incarner en un personnage métonymique la masse, mais bien plutôt d'aboutir à une écriture où l'homme (ou la femme) du peuple s'incarne dans la foule. Des collectifs humain, on ne trouvera donc chez lui ni essentialisation (tradition classique), ni individualisation (tradition contemporaine) ; en termes plus sociologiques, ni approche holiste, ni approche individualiste. C'est bien par les formes du récit, plus souple et plus inventif en ce sens que le carcan académique des historiens, que l'auteur de 14 juillet parvient à dépasser ce paradigme en une Aufhebung qui réconcilie enfin une idéologie progressiste et une écriture révolutionnaire de la masse. Ce faisant, É. Vuillard semble combler un manque dont Jacques Rancière dressait le constat en 1992 :

L'impensable extravagance que l'histoire sociale et ouvrière, à deux ou trois exceptions près, s'est interdit d'imaginer - et qui lui eût été pourtant strictement 
nécessaire -, c'était simplement de se trouver, elle aussi [comme chez Michelet], une poétique. Et sans doute, pour la trouver, fallait-il aller un peu plus avant du côté de la révolution littéraire : là où le roman dit adieu à l'épopée, où la parataxe de coordinations démocratiques succède à la syntaxe des subordinations monarchiques $[. . .]^{23}$.

\section{La liste comme figure totale}

$9 \mathrm{Au}$ cœur du chapitre « La foule », central textuellement et symboliquement dans le récit, le lecteur trouve cette interrogation fondatrice, rappelée en introduction de ce travail: "Qu'est-ce que c'est, une foule? » (p. 84). La réponse semble d'abord décevante: "Personne ne veut le dire ». Mais, en réalité, É. Vuillard apporte un élément de définition fondamental dans la suite immédiate du récit: " une mauvaise liste, dressée plus tard, permet déjà d'affirmer ceci ». La référence aux listes des vainqueurs de la Bastille est évidente ${ }^{24}$, en sorte que cette figure de style est d'abord la marque de la nature des sources du romancier-historien, dont il faudrait interroger la valeur coercitive, puisqu'elle provient, en propre, du pouvoir. Néanmoins, on aurait tort de ne pas voir dans l'utilisation débordante des énumérations une tentative originale pour forger une forme-sens capable d'écrire la foule : si, pour É. Vuillard, il semble que toute définition de ce phénomène social ne puisse être tentée hors d'une poétique particulière, c'est peut-être dans la liste que l'effort définitoire se réalisera ${ }^{25}$.

Cette équivalence absolue entre foule et liste ne doit pas surprendre, si l'on se rappelle la volonté d'É. Vuillard de ne céder ni à l'essentialisation ni au morcellement des foules. De fait, qu'est-ce qu'une liste, sinon une juxtaposition d'items placés les uns à côté des autres sans relation de dépendance et en parfaite horizontalité ${ }^{26}$ ? La liste colle à la foule en rendant compte de la multitude et de la diversité sans prétendre la réduire à un exemplaire ou à une seule de ses dimensions; elle donne à sentir de l'intérieur le vertige du tout sans l'affadir à la partie ; en ce sens, elle se constitue en antisynecdoque $^{27}$ et s'oppose bien à la dynamique d'individualisation qui est la marque de la littérature $\mathrm{du} \mathrm{XIX}^{\mathrm{e}}$ siècle. Transposée au champ politique, c'est une figure que l'on pourrait donc qualifier de radicalement démocratique. Voilà qui explique que le chapitre "La foule ", sorte d'anthropologie ou de phénoménologie vuillardienne, s'apparente à un chapitre-liste qui semble vouloir rendre compte de la bigarrure et de l'étendue de la masse en laissant se succéder les énumérations des prénoms, des noms, des surnoms, des métiers, des âges.

11 On renoncera ici à faire la liste des listes de 14 juillet; tout au plus rappellera-t-on qu'elles essaiment dans le récit comme à partir de ce point central : le lecteur trouve donc au détour de la progression des événements telle ou telle énumération focalisée sur les métiers des gens du peuple (p. 44 ou p. 76), les expressions des émeutiers (p. 104), les surnoms des soldats (p. 122) ou simplement les noms de quelques vainqueurs de la Bastille (p. 138). Il y est même une liste qui, par un habile jeu sur le contenant et le contenu, inventorie les appellations de la foule elle-même ${ }^{28}$. Parler de forme-sens ne paraît donc pas déplacé : par sa symbolique, la liste place dans une catégorie commune des éléments formellement proches, mais dont elle laisse percevoir la diversité ; elle transpose ainsi dans le texte une certaine vision de la foule qui tient de la synthèse entre l'unitarisme de Le Bon ${ }^{29}$ et une approche exclusivement individualiste, puisqu'elle crée une catégorie commune (l'énumération) tout en laissant le champ à des variations stylistiques en son sein, qu'il faudrait au reste analyser plus en détail ${ }^{30}$. De même, dans 
la foule se côtoient des individus qui ont parfois peu à voir les uns avec les autres, mais qui marchent dans le même sens et forment un véritable ensemble ${ }^{31}$. Enfin, une liste, par essence jamais finie, laisse l'imagination compléter ses vides, tout comme une foule pourrait toujours grossir et ne peut se définir par un nombre précis d'individus ${ }^{32}$.

La liste envahit donc le récit chez Vuillard et, du même coup, rend sa dignité formelle à la foule. Néanmoins, les passages qui viennent d'être évoqués ne rendent pas compte d'un certain nombre d'énumérations plus troublantes, qui n'offrent pas de réelle différence structurelle avec les premières, mais qui portent pour autant sur des réalités sociales très éloignées. Comment expliquer en effet l'utilisation d'une liste semblable pour faire le catalogue des objets endommagés dans la " folie Titon ${ }^{33}$ ", nom de la manufacture de Jean-Baptiste Réveillon où commencent les émeutes populaires de 1789 ? Pourquoi l'inventaire, sous la même forme, des délices de Versailles (p. 29), des cartographes de Paris (p. 79), de l'accoutrement d'Éthis de Corny lors de sa tentative de députation (p. 144-145), toutes réalités bourgeoises s'il en est ? C'est que, à notre sens, la liste, depuis le point central que constitue le chapitre "La foule », envahit le récit, le phagocyte et le noyaute, tout comme la foule impose progressivement sa présence à la ville. De ces énumérations, qui sont plus des listes-formes, purs signes disjoints de leur référent, que des listes-sens (comme celles évoquant le peuple), il est sûrement possible de livrer une analyse sociocritique : elles traduisent l'irruption soudaine de la foule et de son principe d'égalité, textualisent ${ }^{34}$ la rupture - ontologiquement politique - avec la phrase, son ordre syntaxique hiérarchisé, ses relations de subordination et de dépendance. En ce sens, l'énergie propre aux masses populaires qui anime le 14 juillet 1789 est illustrée dans les structures du texte par le devenir totalisant de la liste, qui ne vise ni plus ni moins qu'à exprimer la totalité du monde ${ }^{35}$ et à transformer, de l'intérieur, le récit vuillardien : en atteste le fait que la liste prend des proportions de plus en plus importantes, s'allongeant tantôt sur quelques pages, par exemple avec l'inventaire des cadavres de la manufacture Réveillon (p. 21-24), tantôt à l'échelle du livre lui-même, si l'on observe l'enchaînement des chapitres, sur lequel nous aurons l'occasion de revenir.

\section{Le règne de l'indéfini}

13 Nous avons donc vu comment un choix stylistique, loin d'être neutre et de se résumer à de l'habillage, est porteur de sens politique dans 14 juillet. Passant du niveau du style à celui de la langue, nous aimerions insister maintenant, plus brièvement, sur une caractéristique de l'énonciation des foules chez É. Vuillard, à savoir le recours constant à l'indéfini, et essayer de la mettre en relation avec le projet politique de l'auteur. C'est principalement l'utilisation massive du pronom on qui frappe le lecteur du récit et qui semble intimement lié, aux yeux de l'écrivain, à la description du comportement collectif. À bien observer le texte, un motif narratif semble émerger: d'abord la mention générale, extérieure, traditionnelle de la foule, puis l'évocation de ses actions via le pronom indéfini ${ }^{36}$. Encore une fois, il faut souligner ici la cohérence des choix de langue d'É. Vuillard. Dans ce mouvement, qui va de la dénomination générale vers l'indéfini, il y a ce refus de la posture d'extériorisation dont nous venons de parler, la volonté de se mettre au niveau de la masse, voire à l'intérieur d'elle. Il s'agit bien d'écrire « la foule sans nom » (p. 83), expression courante ici remobilisée dans son sens premier - priver la foule du carcan du substantif, en somme. De fait, « par la grâce d'un pronom personnel » comme le relève l'auteur (p. 126), révélant ainsi sa conscience 
aiguë de la valeur des unités linguistiques, il est possible de faire contenir dans le on une véritable " pluralité interne ${ }^{37}$ " sous le sceau de l'indistinction ${ }^{38}$. À l'image de la liste, le pronom renvoie autant à la cohérence et à l'homogénéité profondes de la foule révolutionnaire (et de sa communauté d'intérêt) qu'à sa diversité et à sa bigarrure constitutives: les accords sylleptiques fréquents dans notre texte en témoignent ${ }^{39}$. Cette analyse, que nous limitons ici au pronom on tant elle nous paraît significative, pourrait aussi se mener sur l'utilisation des déterminants indéfinis. On pourrait ainsi faire remarquer une technique de narration, courante dans 14 juillet, qui consiste à présenter un nouveau personnage dans un premier temps sous le signe de l'indéfini avant de lui donner un nom et une histoire ${ }^{40}$. De cette façon, l'individu apparaît d'abord comme un au milieu de la foule, et l'individualisation qui suit ne présente pas le caractère d'un portrait hors sol.

Le on paraît donc tout à fait idoine à incarner la foule d'É. Vuillard, à adopter son point de vue médian et son refus de l'extériorisation. Mais ce pronom acquiert aussi une légitimité en quelque sorte extérieure dans le projet politique de l'auteur de 14 juillet. Son caractère indéfini ne marque pas les masses à qui il réfère, ne les circonscrit pas à leur contexte, les émancipe du cadre chronologique de la Révolution : il est vecteur d'universalisme. Nous aurons à revenir sur l'extension du propos politique de Vuillard au-delà de la simple prise de la Bastille, notamment à la fin du récit, et à son arme principale, l'anachronisme ; contentons-nous ici de remarquer que l'utilisation du on pour désigner la masse des dominés depuis laquelle "il faut envisager les choses " (p. 83) se retrouve dans La Bataille d'Occident (2012) pour narrer les actes de la soldatesque ${ }^{41}$. Le on constitue ainsi une passerelle entre des foules de natures diverses dont il s'agit de réévaluer le rôle historique ; et cela est rendu précisément possible par l'indéfinition du pronom, qui fait écho à l'anonymat constitutif de la foule ${ }^{42}$. C'est d'ailleurs ce pont entre les différentes masses de l'Histoire qui permet le glissement jusqu'à l'époque qui est la nôtre. Celui-ci s'effectue grâce au passage du on au nous, par exemple dans la description de Paris qui « est désormais au peuple» (p.48): «On danse autour du monde qui se renverse, le regard se perd dans le feu. Nous sommes de la paille » (p. 49) ${ }^{43}$. De fait, une lecture hors contexte de tel ou tel extrait placé sous le signe du on pourrait sans peine s'appliquer aux dominés d'aujourd'hui (p. 151) : «Bien sûr, ce n'est pas facile tous les jours. On n'a pas encore payé son terme, le mois a été dur, peu de travail et de la dépense. Mais on se serre les coudes. On est jeune, on espère ».

\section{Le récit de 14 juillet : composer avec la foule}

15 Si c'est dans les ressources de la littérature et du travail stylistique que l'auteur puise une représentation historiquement plus juste de la foule, il convient d'examiner comment le collectif révolutionnaire trouve sa place dans le genre textuel qu'É. Vuillard inscrit au fronton de son œuvre. Changeant donc d'échelle, nous passons à présent du niveau de la phrase à celui de l'« architexte $^{44}$ ». Après Conquistadors (2009), œuvre présentée comme un roman, 14 juillet appartient à une succession de livres portant tous la mention " récit ». Cette distinction témoigne d'une conscience à l'endroit des modèles textuels et génériques ainsi que des interrogations qu'ils portent. Force est de constater que le roman est la forme littéraire dominante depuis le $\mathrm{xx}^{\mathrm{e}}$ siècle, mais cette étiquette s'impose souvent par défaut pour les œuvres fictionnelles en 
prose. La préférence pour le "récit " se comprend comme l'intention de relater un événement au ras de l'Histoire, et, partant, de seconder l'historien, dont le travail consiste à interpréter des sources et à disposer ses observations dans un ordre temporel circonscrit. Dans Temps et Récit, Ricoeur situe la similitude de l'activité de l'écrivain et de l'historien sur le terrain du récit, soit la mise en intrigue temporelle à partir d'une expérience du temps foncièrement hétérogène, donc de la compétence narrative ${ }^{45}$. Néanmoins, le choix affiché de la catégorie "récit » n'efface en rien le recours aux astuces et atouts du roman, comme nous le verrons plus loin ; l'ambivalence demeure, et c'est là que réside sa richesse. Le titre 14 juillet pose une inscription nette dans le temps chronologique, mais Vuillard s'attache davantage à représenter le temps vécu par la foule des insurgés, dans lequel le narrateur se projette à corps perdu et puise les éléments qui serviront à la mise en forme temporelle, esthétique et énergétique du récit. C'est contre une multiplicité de récits de la prise de la Bastille, jugés insuffisants voire pleins de suffisance, que s'écrit 14 juillet - récit, dont l'injonction paradoxale, «il faut écrire ce qu'on ignore » (p. 83) trouve son objet dans " la foule sans nom » (p. 83). Puisque récit il y a, observons donc la place que la masse occupe dans la narration.

\section{Instruire et épouser le mouvement de la foule : la construction cyclique de l'œuvre}

16 L'insurrection populaire ouvre et clôt le récit. Le premier chapitre raconte le saccage de la folie Titon, qui se solde par un échec, tandis que l'ultime chapitre, «Pluie de papier », célèbre le moment où la foule s'empare de la Bastille et rachète la frustration inaugurale. Le succès de la prise de la Bastille, attendue, bénéficie ainsi d'un intérêt narratif bien différent du suspense. Pour les deux événements, le narrateur invite le lecteur à partager la joie avec la foule: "Que c'était bon! [...] Le produit dérobé du travail doit être gaspillé, sa délicatesse meurtrie » (p. 16), "Quel bonheur de caillasser les argousins !» (p. 17), «Que c'est bon de défaire et de démolir! Personne ne pense à demain. On désire tout renverser, tout jeter, sacquer, révoquer, flanquer par terre ! Et ça fait plaisir, un plaisir inouii. » (p. 195) Le récit cède au discours, mimant l'exaltation par le recours à des phrases exclamatives et en multipliant des verbes synonymiques pour traduire le sentiment d'une puissance retrouvée. La saisie de ces lieux qui symbolisent l'injustice est dépeinte comme une fête libératrice (p. 17) : « Ils voulaient faire chanter les lustres, ils voulaient danser parmi les voilettes, mais surtout, ils désiraient savoir jusqu'où l'on peut aller, ce qu'une multitude si nombreuse peut faire. » Si le lecteur avisé sait jusqu'où les foules du XviII siècle finissant sont allées et n'est donc guère tenu par le suspense, du moins participe-t-il par le truchement du texte à un temps suspendu à la jouissance de l'instant. Ce désir d'interruption est d'ailleurs attisé par ces groupes verbaux au présent que l'auteur souligne : voilà des pensées-actions, vraisemblablement subconscientes et collectives, qui actualisent l'expérience-limite provoquée par le flux insurrectionnel dans le for intérieur de l'individu, confronté soudainement et paradoxalement à sa propre puissance au contact de la foule, mouvement complexe traduit par la simplicité du passage sans heurt du pluriel au singulier. C'est de fait ce déploiement de puissance et d'action qui charpente le récit d'É. Vuillard. 
17 L'expérience-limite implique également le péril de mort. Les morts sont moins nombreux lors de la prise de la Bastille que lors du saccage de la folie Titon, mais la foule de disparus trouve également sa place dans la composition du récit. En effet, le second chapitre, «La Tombe-Issoire ", est consacré à l'autopsie légale d'une vingtaine d'émeutiers, dont l'inventaire souligne la similitude, sinon l'égalité. À la fin du chapitre, la famille Petitanfant est appelée à confirmer l'identité de leur parent défunt (p. 28) : « Ils ont reconnu leur frère, lequel se nomme Augustin Vincent Petitanfant ; il était âgé de vingt et un ans, il était manœuvre et maçon et il demeurait avec ses frères. Après quoi, le clerc releva la tête, et leur demanda de bien vouloir signer le document. Ils ne savaient pas écrire. » (p. 28) C'est à travers de telles mises en scène fictives du monde administratif que Vuillard entend démontrer comment les acteurs des événements échappent aux archives et, partant, aux sources des historiens. Le lecteur s'associe aisément à cette position tant le gouffre entre l'administration et l'existence demeure une expérience familière. Cette scène entre en écho avec la fin de l'avant-dernier chapitre, « Le déluge ", où « Marie Jeanne Bliard, veuve de François Rousseau, allumeur » (p. 192), ressent, au milieu de ses souvenirs du défunt, un malaise face au traitement indifférent et indigent du commissaire de police (p. 192) : «Elle eut un frisson. Ses lèvres se raidirent. Elle releva la tête, fixa terriblement l'homme qui se tenait en face. Il ne la voyait pas. Il écrivait. » É. Vuillard fustige ainsi une écriture aveugle au profit d'un récit qui regarde les êtres dans la foule, opération qui sollicite ou bien toute une gamme d'affects, allant du comique au pathétique, ou bien l'intelligence du lecteur à voir au-delà des mots ${ }^{46}$.

Le début et la fin du récit sont donc construits en miroir, moments suspendus de fête et de mort, encadrant et rehaussant les péripéties qui ont débouché sur la prise de la Bastille. Entre ces deux moments, le cadre spatio-temporel est resserré, concentré, à l'image de la densité de la foule. Tandis que les deuxième et troisième chapitres prennent le soin de rapporter la succession des événements politiques des mois précédant le 14 juillet, le récit est progressivement envahi par la foule, notamment à partir de l'afflux des provinciaux, de la même façon que nous avons vu que la langue était progressivement contaminée par la liste. L'expansion de la foule motive le narrateur à légitimer son récit par un propos métapoétique fondé sur l'ignorance et la célébration de l'ambivalence du langage ${ }^{47}$ :

Ah ! nous ne pourrons jamais savoir, nous ne saurons jamais quelle flambée parcourut les cœurs, quelle joie ; nous pourrons peut-être brûler du même feu, mais pas le même jour, pas la même heure, nous pourrons bien interroger minutieusement les mémoires, parcourir tous les témoignages, lire les récits, les journaux, éplucher les procès-verbaux, on ne trouvera rien. La véritable pierre de Rosette, celle qui permettrait d'être partout chez soi dans le temps, nous ne l'avons jamais trouvée. La vérité passe à travers nos mots, comme le signe de nos secrets. (p. 64-65)

Le pronom inclusif nous assure la cohésion de ce passage et tente d'emporter l'adhésion du lecteur au projet littéraire d'É. Vuillard ; le on d'un historien soucieux d'exactitude est renvoyé à ses limites. Certifiant par le présent gnomique l'incertitude fondamentale qui régit le rapport à l'autre, a fortiori à une foule, le narrateur propose ici un pacte de lecture : à défaut de certitude, le langage peut (en puissance) recouvrir cette "vérité » et ces "secrets ". C'est au nom de ce potentiel véridictionnel du langage qu'est également justifié le recours aux anachronismes, car « à travers nos mots " (nous soulignons), le narrateur désigne notre langage contemporain aussi bien que le lexique d'époque, consigné dans ce que l'on a l'habitude d'appeler le trésor de la langue. Ce 
faisant, le récit s'approprie ce qui passe encore, malgré l'écart temporel, entre le passé et le présent. De surcroît, l'effacement de l'année 1789 dans le titre institue le soulèvement des foules dans un cycle anthropologique calendaire, au-delà de toute chronologie, où la saison estivale reflète l'ardeur des aspirations collectives. L'ordre du récit de 14 juillet a beau être romanesque, progressant d'une situation initiale vers un dénouement, c'est dans la mise en forme d'une syntaxe qui mêle les temporalités que s'échafaude un récit créant un continuum entre le temps narré et le temps de la narration, tant par le choix du lexique que du temps verbal ${ }^{48}$.

Il semblerait en somme que le récit procède, à l'image de la métaphore récurrente du feu, de la flambée, de la paille qui s'embrase, d'un déploiement rapide de forces naturelles qui se consument mais se propagent, de la foule comme une densité humaine parcourue par une énergie qui jaillit çà et là, sur laquelle le narrateur se focalise tour à tour selon son intensité. Le récit, net dans son cadre spatio-temporel, rétif à l'écueil de l'essentialisation et de la psychologisation individuelle, tente alors de conjuguer la force massive de la foule et le surgissement spontané des actions, défi technique et artistique majeur qui puise sa force dans l'instabilité même de la situation.

\section{Un récit fort de son instabilité : le moment insurrectionnel, entre épique et lyrique}

21 La foule assiège la Bastille, " citadelle ", «temple d'Horus » (p. 63), protégée par des soldats armés, surplombant les lieux. Le rapport de force est tendu, instable, les coups de feu venant des tours dispersent tant bien que mal les insurgés, comme au début du chapitre « Le pont-levis» (p. 121). À cette situation bloquée, É. Vuillard fait succéder une action qui puise largement dans le registre épique, propre à exalter les gestes d'un héros, et dans le registre lyrique ${ }^{49}$. Le narrateur introduit cette séquence épique par un (nouvel) aveu d'ignorance, comme pour se défendre de pratiquer un romanesque involontaire (p. 123-124) : « À partir de ce moment, on ne comprend plus rien. Les lieux vacillent, le temps meurt. Tout se précipite. [...] Jean-Armand Pannetier [...] met le feu aux poudres » : laissant derrière lui « une petite relation de sa journée », il encourage " le charron Tournay » à escalader les bâtiments attenants pour détruire le mécanisme du pont-levis. Tournay devient, selon le désir du narrateur (qui déclare "je désire ", p. 124, en son nom propre) " lui-même, seulement lui-même, vraiment, dans son intimité la plus parfaite ", après être monté sur les toits, « aux yeux de tous » (p. 124). Le héros épique se distingue par ses accomplissements ; s'il n'existe que dans son action, dans ses hauts faits, il requiert néanmoins un spectateur. Ainsi se justifierait la focalisation sur un personnage, devenant tel en vertu de son geste, ou de sa geste, aussi brève fût-elle. Cette grandeur, le narrateur la suscite en décrivant « quelques pas de danse sur un toit de tuiles. Une série de déboulés, la tête libre, haute ", qu'il corrige dans la phrase suivante: "ou plutôt, non, ce furent des pas très lents, de petites glissades, des pas de chat » (p. 124), épanorthose qui souligne le trouble du narrateur, oscillant entre l'exaltation du conteur saisi dans son acte et la réserve sobre du récit réaliste. Aussitôt, le récit prend un tour lyrique, visant à émouvoir le lecteur, motivé par la soudaine solitude de Tournay, dans la " cour du Gouvernement » (p.125): «Personne ne sait de quoi la liberté est faite, de quelle façon l'égalité s'obtient. Louis Tournay, le charron, le jeune homme de vingt ans, est passé de l'autre côté de la vie. Il n'est pourtant qu'un petit morceau de foule tombé là, tout seul. » La grandeur de Tournay est paradoxalement soulignée par sa petitesse ; nous sommes dans le sublime. 
D'autres insurgés suivent son parcours, comme pour désindividualiser le récit (p. 126) : "Il faut être attentif à ces vagues présences, contours, profils, à ces locutions dont tout récit se sert pour mener son lecteur. Gardons-les encore contre nous un instant, ces huit à dix autres, par la grâce d'un pronom personnel, comme de tout petits camarades. "L'intrication entre le récit de la foule et les commentaires méta-narratifs peut être interprétée de bien des manières. Dans tous les cas, elle souligne la faculté aussi bien autoréflexive qu'émotive du récit. É. Vuillard renonce au récit où, selon les mots de Benveniste, "les événements semblent se raconter eux-mêmes ${ }^{50}$ ", mode de narration décidément impossible, sinon inadéquat, pour représenter l'insurrection populaire, dont l'intensité des passions et la conscience momentanée d'un ensemble supérieur à l'individu excèdent d'une part l'événement historique en tant que tel, son historiographie, et, d'autre part, tout auteur-scripteur, qui, à moins de servir un discours en particulier, ne peut être qu'un individu.

14 juillet puise son énergie justement dans l'implication du narrateur dans le récit, dans la sollicitation des affects du lecteur, affects qui se rejoignent et éclatent dans la matière textuelle. Les glissements constants entre récit et discours, personnages et narrateur, indétermination et réception génèrent décidément une narration qui fait la part belle à l'instabilité, à la contingence, ce qui est loin de nuire au projet de cette œuvre. En effet, elles ne se font guère ressentir comme des obstacles à la lecture, mais bien comme des garanties d'un récit vivant et mouvant, s'opposant à un récit monolithique et pétrifié. La gravitas de l'historien et la solennité de l'événement sont bien déjouées lorsque la foule s'apprête à entrer dans la Bastille, avec cette fin de chapitre « Les funambules » qui relève du burlesque (p. 178) : « La Bastille était devenue une simple maison à la porte de laquelle le monde frappait. Alors, scène irréelle, comme le portier de nuit qu'on réveille dans un hôtel et qui bâille, un invalide, ignorant tout de la rhétorique des grandes occasions, entrouvrit et demande poliment ce qu'on voulait. " Par ces fréquents décrochages de registres, 14 juillet met en lumière le caractère protéiforme de la réalité, et suggère combien celle-ci peut faire écho aux registres divers de la fiction, sans qu'aucun ne puisse prétendre à une autosuffisance ou à une supériorité. La polémique avec l'historiographie traditionnelle de la Révolution française est de cette façon relancée, implicitement au moins. Ainsi, une fois que Louis Tournay a mené à bien son entreprise exceptionnelle, hérö̈que, " on l'oublie. Il s'évapore. Son épopée n'aura duré que quelques minutes. " $^{51} \mathrm{~A}$ la foule de la Bastille répond donc toute une gamme de registres qui font le lien entre l'événement narré et le lectorat, soulignant par leur instabilité la contingence des réalités humaines. C'est pourquoi il convient de s'interroger sur le discours qu'É. Vuillard porte sur le potentiel d'action de la foule, discours qui, en vertu des nombreux anachronismes qui actualisent l'événement, est nécessairement pénétré d'une réflexion contemporaine sur la démocratie et les mouvements de contestation. Quelles vertus politiques - actuelles ? l'auteur prête-t-il à cette masse?

\section{4 juillet et son propos : discours et image de la foule}

Ainsi l'écriture de la foule dans 14 juillet se donne-t-elle comme ambition de dire la foule tout en refusant une hiérarchisation claire : celle qui accorde à un mot le privilège de porter le sens de la phrase (mots ici mis en équivalence dans la liste), celle qui accorde à un personnage, à un point de vue, le privilège de guider l'attention (points de vue ici 
mis en équivalence dans la foule des acteurs), celle qui accorde à une intrigue le privilège de guider l'histoire (intrigue ici mise en équivalence dans la multiplicité des scènes). Cette hiérarchisation ne semble toutefois refusée que dans la mesure où elle est inadéquate au projet de raconter la foule (projet lui-même aporétique pour É. Vuillard $)^{52}$. Il n'en demeure pourtant pas moins qu'un certain nombre de discours traversent le récit, qui contribuent à façonner une certaine image de la foule. Et il n'est pas sûr, ici, que la mise en équivalence soit de mise. En effet, à travers les phénomènes de brouillage énonciatif du narrateur, qui tantôt se manifeste explicitement (à la première personne du singulier), tantôt s'inclut dans la foule, tout en gardant une distance dans l'indistinction (par le pronom on), tantôt s'associe nettement au peuple par une fiction d'unité (le nous, dès la page 4), tantôt, enfin, généralise à partir de formules impersonnelles et des présents de vérité générale, É. Vuillard maintient un discours visant à dire le soulèvement de la foule, et ce avec elle, au sens poétique et politique, c'est-à-dire partisan du terme. Cela passe d'abord par une prise de distance ironique vis-à-vis des discours sur la foule (ceux des acteurs historiques notamment, et des préjugés sur lesquels ils reposent) et leur mise en tension avec un autre discours, plus enthousiaste, les deux allant parfois de concert dans ce qui s'apparente à de nets commentaires. Mais ce premier renversement demeure peut-être encore trop formel ou, du moins, comme la foule elle-même qui ne peut supporter d'être distinguée en éléments individuels sans être par là dissipée, il est inséparable, comme enjeu poétique, d'un enjeu politique. Ainsi, la colère et la jubilation, mais la peine aussi, sont à la fois des éléments moteurs de la narration et l'enjeu d'une mise en mouvement, d'une émotion, d'une dynamique qui transporte auteur, récit et lecteur ${ }^{53}$. Cette dynamique provient d'éléments sociaux et politiques que sont la faim, l'exploitation, l'humiliation, la répression, le soulèvement, la destruction. Le récit, dès lors, porte moins sur la finalité de l'histoire que sur les possibilités de sens immanentes à la dynamique des gestes qu'il raconte et qui va jusqu'à nous.

\section{Un travail de sape des gestes et discours hostiles : vers une légitimité de la foule}

Dès ses premières pages, 14 juillet porte son attention sur le peuple mis en mouvement qu'est la foule. Parallèlement, il envisage le discours sur cette foule, les gestes et actions vis-à-vis d'elle. Puisque le récit d'É. Vuillard ne se propose pas d'être un travail d'histoire, même s'il s'agit d'un récit historique, le rappel de ces éléments n'équivaut pas à la présentation de causes, ou même, littérairement, aux motivations nécessaires à la vraisemblance de la narration. Ces éléments font partie intégrante du projet de raconter le soulèvement de la foule: ils en déterminent l'image et sont un espace d'engagement polémique. Dès les deux premières sections du récit, « La folie Titon » et «La Tombe-Issoire », on relève cette lutte. Elle passe d'abord par des contrastes: la folie Titon, c'est la demeure des riches, du propriétaire, Jean-Baptiste Réveillon; la Tombe-Issoire, c'est la rue au-dessus des catacombes, la demeure des pauvres. À l'« extravagance d'architecte » (p. 9) de la folie Titon répondent les « dédales sinistres » (p. 20) des catacombes. Plus encore, si les listes de la première section visent à signifier l'opulence de la folie Titon, la liste de la seconde (les cadavres numérotés sont examinés un par un), par la répétition des mêmes éléments (anaphore d'« encore ", p. 23), indique la pauvreté unifiante des ouvriers-émeutiers. Ce contraste repose par ailleurs sur un élément de discours mis en valeur par É. Vuillard : si Jean-Baptiste Réveillon a 
cherché à réduire les salaires, c'est notamment parce que certains de ses ouvriers seraient déjà " riches ", avec « la montre dans le gousset » (p. 10). L'italique, dû à l'auteur, vient ici signaler la prise de distance avec ce propos qui indique la simplicité de cette possession, comparée au luxe que Réveillon manifeste, et une mise en évidence qui permet de créer une continuité du récit, puisqu'on retrouve la même expression plus loin. En effet, à l'issue de la fouille des cadavres, qu'on soupçonne d'avoir volé dans la folie Titon, il n'y a " pas la moindre montre dans le gousset » (p. 24). Ainsi, non seulement il n'y a pas eu de vol, mais les ouvriers n'ont même pas cette fameuse montre qui devait témoigner de leur enrichissement ${ }^{54}$. Cette image de l'ouvrier-émeutier justifie d'une part sa répression, d'autre part son humiliation et si d'un côté tout est compté, (« tout cela fit l'objet d'un compte précis ", p. 19), de l'autre, «le nombre de morts [...] reste vague, indécis ${ }^{55} »($ ibid.). Ainsi apparaît clairement l'axiologie, plutôt explicite, du rapport à la foule: celle de la présentation par l'auteur d'une constante méprise, doublée d'un mépris constant.

On la retrouve dans la parole citée des chroniqueurs et historiens. On repère ainsi deux exemples seulement de parole directement citée: la première est celle d'un chroniqueur de l'époque, Galort de Montjoie, rapportée par Hippolyte Taine dans Les Origines de la France contemporaine, où la Révolution est présentée dans une perspective très négative, celle de "l'anarchie ${ }^{56}$; la seconde est tirée du chapitre consacré à la prise de la Bastille par Jules Michelet, dans son Histoire de la Révolution française (p. 99 de 14 juillet). Dans le premier cas, il s'agit d'une description des bandes de vagabonds, brigands, qui, rapidement, se sont mêlés aux foules révolutionnaires. Sans mettre particulièrement en cause leur description, É. Vuillard semble placer à distance, par le recours à la citation, ces brigands qui, de toute évidence, ne l'intéressent ni ne l'émeuvent beaucoup. Le second cas, sans ironie, marque cependant une distance beaucoup plus grande vis-à-vis de Michelet. Si son talent d'écrivain est reconnu, sa réduction de la prise de la Bastille à l'œuvre d'un seul homme ${ }^{57}$, Thuriot de la Rosière, semble pour l'auteur procéder à un véritable effacement de la foule au profit d'un représentant. Pour mieux faire sentir ce grief, après l'avoir énoncé, É. Vuillard procède à la description de Thuriot quittant la Bastille, le mettant aux prises avec la foule : « on lui arrache sa chemise »(p. 102) $)^{58}$. Thuriot est ainsi décrit comme sourd, par trop d'individualisme : « comme bien des parlementaires après lui, il n'écoute pas, il ne saisit pas ce que veut cette foule » (p. 102-103). Lorsque ce dernier s'extirpe enfin de la foule, É. Vuillard évoque non plus le contraste entre ce noble élégant et la foule sale et sauvage, mais sa défiance à l'égard d'un peuple qui lui reste incompréhensible.

Mais il ne suffit pas à É. Vuillard de souligner les abus de langage de ceux qui parlent de la foule, les subterfuges par lesquels, en dernier lieu, on fait disparaître leur présence envahissante, à tous les sens du terme. Il met aussi en avant une parole bien spécifique. Elle est double, et référencée, mais pas citée. D’abord celle de Mirabeau (p. 43) : «Oui, Mirabeau parle. Il est un sentiment, une vérité. Nul ne peut plus rien contre. Il dit. La grosse gueule s'ouvre pour la première fois avec autant de souffle et de culot. La volonté du peuple vient de faire son entrée dans l'Histoire. » Mais s'il parle, et que cette parole s'identifie à la volonté du peuple, c'est que les mots le possèdent, plus que l'inverse. Cela est confirmé quelques pages plus loin avec les mots de Camille Desmoulins, un jeune avocat :

Les petits mots de Camille ricochent partout, ils pissent, ils suintent, ils sont la

forme de ce monde ; comme ceux de Mirabeau, ils touchent à une matière sans preuve, un stigmate, une foi ; loin du menuet du langage, ils sont un signe, 
compréhensible à tous et pourtant insondable; ce sont les mots de tout le monde.

(p. 47) mais par quelque chose qui nous attire vers la foule, une « autre chose de plus difficile à nommer, de plus impossible à rater, de plus jubilatoire » (p. 72). Cette chose échappera à la nomination ${ }^{59}$, comme la foule aux recensions historiques, mais elle détermine une continuité possible entre cet événement et nous. Cette continuité se marque dans le discours par deux phénomènes: une extension de l'événement qui finit par nous inclure et un rapprochement qui finit par nous superposer. Le premier phénomène se manifeste par une généralisation dans les remarques portant sur la foule. On en relève une dizaine de quelque importance ${ }^{60}$. Elles contribuent tout d'abord à rendre à la foule la légitimité de son expérience en accordant à ses désirs une valeur positive. Encore une fois, Thuriot sert de repoussoir :

Il n'imagine pas que la multitude puisse savoir quelque chose, désirer quelque chose, avoir raison même, et qu'après tout, c'est bien elle le souverain, ce sont ces bonnes femmes qui jacassent, ces crétins qui crient, ces gens qui l'empoignent et exigent des comptes. (p. 103) elle-même sur le modèle de ceux qu'elle renverse : sa parole est bien confuse et profuse, excessive en quantité et en volume, d'une traite avec le geste. De plus, elle fait irruption par son exigence : elle demande qu'on en tienne compte. La foule représente ainsi une puissance, de désir et d'action : "ils désiraient savoir jusqu'où l'on peut aller, ce qu'une multitude si nombreuse peut faire "(p. 16). Où l'on voit deux choses: que lorsqu'É. Vuillard parle de la foule dans sa légitimité publique, il a recours au terme de multitude, un terme qui a une histoire politique ${ }^{61}$, et que, par ailleurs, il insiste sur son désiri ${ }^{62}$. On en trouve d'autres mentions : « on avait le désir d'un autre corps » (p. 54-55), « il croit en ce qu'il désire » (p. 119), qui recoupent le désir du narrateur lui-même : « je désire, j'imagine » (p. 124). Et si ce désir s'étend jusqu'à nous, c'est par une sorte de loi de l'intermittence des soulèvements (p. 60): «Ainsi, la sédition. Elle surgit dans le monde et le renverse, puis sa vigueur faiblit, on la croit perdue. Mais elle renait un jour. Son histoire est irrégulière, capricante, souterraine et heurtée. " C'est cette intermittence ${ }^{63}$ qui permet l'appel final à reproduire le geste de dispersion des papiers et qui fait dire à É. Vuillard que si « nous ne saurons jamais quelle flambée parcourut les cœurs, quelle joie [...], nous pourrons peut-être brûler du même feu, mais pas le même jour, pas la même heure " (p. 64). La révolte dispose donc d'une continuité directe par une unité de désirs, en revanche, dans sa forme, elle ne peut qu'être différente et justifie ainsi les précautions de l'appel final, écrit au conditionnel.

Une fois mis à distance le fantasme d'un savoir exact du passé, le récit proposé peut envisager une plus grande liberté d'écriture. On remarque ainsi un emploi récurrent de 
l'anachronisme, ainsi désigné par référence au cadre général, mais non revendiqué, d'un récit historique, daté. Le texte regorge de termes ou d'expressions qui rabattent soudainement l'action décrite sur notre période contemporaine. Dès la page 12 , on relève : «Un peuple de chômeurs aussi. " Si le chômage existe depuis longtemps, et encore pas dans le sens actuel, le chômeur, lui, est récent ${ }^{64}$. Plus loin, Necker, le fameux banquier, est comparé aux " traders qui, de nos jours, jettent leurs ordres entre les mâchoires du monstre, en espérant que cela passe » (p. 39). Et si les dirigeants de l'époque sont rapprochés des privilégiés d'aujourd'hui, alors les insoumis d'hier peuvent ressembler aux nôtres (p. 48) : «Pour se défendre, les gens improvisent des barricades de chaises, puis ils se saisissent de bâtons, de caillasses, et c'est l'intifada des petits commerçants, des artisans de Paris, des enfants pauvres. »

31 Ainsi, ces brusques contractions de l'espace-temps par le langage nous engagent dans une actualisation de la situation, comme É. Vuillard le dit d'ailleurs directement dans son texte de présentation : « À une époque où un peuple se cherche, où il apparaît sur certaines places de temps à autre, il n'est peut-être pas inutile de raconter comment le peuple a surgi brusquement, et pour la première fois, sur la scène du monde ${ }^{65}$. "

\section{NOTES}

1. Éric vUILLARD, 14 juillet, Arles, Actes Sud, 2016, p. 84. Par la suite, lorsqu'un numéro de page sera cité entre parenthèses, c'est à cette œuvre que nous renverrons.

2. «C'est depuis la foule sans nom qu'il faut envisager les choses» (p. 83)

3. Voir ainsi : « Nul n'a jamais écrit leur fable amère » (p. 33); « et l'on doit raconter ce qui n'est pas écrit » (p. 83).

4. On peut citer ici un entretien entre Éric VUILLARD et Pierre SCHOENTJES, « Dialogue : de l'Histoire à la littérature ", dans Gianfranco Rubino et Dominique Viart (dir.), Le Roman français face à l'Histoire: thèmes et formes, Macerata, Quodlibet, 2014, p. 275-286. Voir aussi la recension de 14 juillet de Laurent DEMANZE, "Émeutes historiographiques selon Éric Vuillard (14 juillet)», Diacritik, $1^{\text {er }}$ décembre 2016, disponible en ligne : https://diacritik.com/2016/12/01 (consulté le 07/01/2020). Cf. également une émission de France Culture, Dans les coulisses de l'Histoire avec Éric Vuillard, du 17 mai 2017 (27 minutes).

5. Fournier, quittant la « foule » qui s'approche de la Bastille (p. 66) pour mettre un enfant blessé à l'abri, rencontre en s'éloignant des "gens " qui « s'approchent timidement " (p. 68). Ce substantif sert aussi à caractériser les bourgeois de l'Hôtel de Ville, tout à fait distincts du mouvement révolutionnaire ( "les gens de l'Hôtel de Ville souhaitent qu'on retire les canons afin de calmer les esprits ", s'opposant ainsi au «peuple », p. 98 ; les tournures euphémisantes semblent aller de pair avec l'emploi du terme de gens). Cf. aussi p. 105.

6. L'articulation groupes-foules se constate par exemple dans l'emploi des temps : p. 64, « toute la nuit, des groupes s'étaient formés sous les tours ", action antérieure (plus-que-parfait) et menant à la composition d'une foule (« une foule [...] errait autour des murailles », imparfait). Cf. aussi p. 54 .

7. Voir l'épisode de la manufacture Réveillon (p. 16-17): la scénographie nous fait voir, à l'intérieur du bâtiment, " une foule d'hommes » qui « arrach[e] aux entrailles de la terre 
quelques bouteilles » (verbe d'action) ; à l'extérieur, « il y avait une masse de trente mille curieux » (simple état, sans action ou revendication propre).

8. Georges Lefebvre, «Foules révolutionnaires ", dans Études sur la Révolution française, Paris, PUF, 1954 (première publication 1934), p. 271-287, où l'historien proposait de parler d'agrégat et distinguait dans cet ensemble l'« agrégat involontaire et éphémère d'individus » (p. 273), les « agrégats semi-volontaires » (p. 274) et, enfin, « l'état de foule » (p. 277). Les premiers semblent correspondre aux groupes ou aux gens d'É. Vuillard, les seconds aux masses, les derniers portent, comme dans 14 juillet, le nom de foule.

9. « Camille propose au peuple la colère. [...] La foule tombe d'accord. Les paroles du jeune homme font écho à nos peurs, à l'inquiétude qui monte, au manque de pain » (p. 46, remarquer l'emploi de la première personne du pluriel). Se structure ainsi une opposition dont les deux pôles sont la pensée politique et la réalité sociale.

10. Georges RUDÉ, La Foule dans la Révolution française, Paris, François Maspero, 1982 (édition originale 1960).

11. Sur la déconstruction de l'image de la foule élaborée par l'historiographie contrerévolutionnaire, voir Philippe MÜNCH, «La foule révolutionnaire, l'imaginaire du complot et la violence fondatrice : aux origines de la nation française (1789) ", Conserveries mémorielles, $\mathrm{n}^{\circ} 8$, 2010 (avec des références complémentaires).

12. Sur la question, on lira l'étude synthétique de Thomas BAIER, «Die Darstellung der Masse in der römischen Literatur ", Aevum, 84, 1, 2010, p. 161-176, qui insiste sur cette indifférenciation et cette perspective surplombante des auteurs latins, du moins jusqu'à l'Antiquité tardive. Pour une synthèse récente de l'historiographie de la notion de foule, en particulier en sciences de l'Antiquité, voir Louis AUTIN, Voix de la foule chez Tacite. Perspectives littéraires et historiques sur la communication collective au début de l'Empire, thèse de doctorat (Université Grenoble Alpes, Universität Osnabrück), p. 19-40.

13. Politique, III, 11, 1281a-b notamment. L'éloge de la bigarrure de la foule est un des traits qui reconduisent Vuillard au Stagirite ; ainsi peut-on lire page 90 de 14 juillet, dans le tableau de la foule révolutionnaire et $\mathrm{du}$ « bonheur » qu'elle procure : « tous les accents se mêlent, les patois, les métiers".

14. Selon la célèbre expression de la République, VI, 493a-d.

15. Voir notamment Jean-Pierre VERNANT, «Le Moment historique de la tragédie », dans JeanPierre Vernant et Pierre Vidal-Naquet, Mythe et Tragédie en Grèce ancienne, Paris, François Maspero, 1972. Sur la question, voir la synthèse récente de Claude CALAME, La tragédie chorale: poésie grecque et rituel musical, Paris, Les Belles Lettres, 2017. Qu'Éric Vuillard cherche à dépasser ce traitement de la foule sous l'angle du personnage collectif, cela est évident dans la description de Paris, notamment page 77, où l'affirmation initiale (" une ville est un personnage ») est contredite par la suite directe (« pas de vaudeville ou de tragédie, non, un personnage pour pièce en plein air, sans figurant, sans chœur, sans mise en scène ", nous soulignons) et la liste qui vient juste après.

16. Jules MICHELET, Histoire de la Révolution française, Paris, Chamerot, $2^{\mathrm{e}}$ édition, 1893-1898 [1 $1^{\mathrm{re}}$ édition 1847], livre I, chapitre VII, récit de la prise de la Bastille où Michelet célèbre « l'instinct vengeur du peuple» (p. 239) ; Hippolyte TAINE, Les Origines de la France contemporaine, III, La Révolution : la conquête jacobine, Paris, Robert Laffont, 1986 [1 ère édition 1881], p. 239.

17. Gustave LE BON, Psychologie des foules, Paris, Félix Alcan, 1895. Sur Le Bon, Tarde et leurs successeurs aussi bien que leurs précurseurs, l'ouvrage de référence reste l'étude de Susanna BARROWS, Miroirs déformants : réflexions sur la foule en France à la fin du XIXe siècle, Paris, Aubier, 1990, trad. de S. Le Foll (édition originale 1981).

18. «La volonté du peuple vient de faire son entrée dans l'Histoire », p. 43. 
19. Comme le rappelle Thomas Pavel à propos de Stendhal (Thomas PAVEL, La Pensée du roman, Paris, Gallimard, p. 260-261). Le " procès en picaresque » fait précisément partie de l'instruction à charge que É. Vuillard adresse au roman via l'œuvre de Manzoni, comme expliqué lors de la conférence du «Banquet d'Automne » de La Maison du banquet et des générations (disponible en ligne https://www.youtube.com/watch?v=eSUsgyy06Gs, consulté le 07/01/2020).

20. Décrivant Paris et listant les noms de certains de ses habitants, É. Vuillard conclut sans aucune forme d'ambiguïé : « mais bientôt on aura un nom, on s'appellera Étienne Lantier, Jean Valjean et Julien Sorel » (p. 78, nous soulignons). Le refus de l'individualisation est évident. L'âge où un auteur peut commencer son œuvre par " je suis né d'une famille pauvre » est ainsi postérieur à la Révolution (p. 117).

21. Ainsi, cette tentation reste cantonnée aux souvenirs, ceux de Louis et Louise Petitanfant (« ils s'étaient construits de minuscules cabanes en face de chez eux [...]. C'étaient trois huttes minuscules, faites de pierres roulées, de boue et de vieilles planches »), ceux de Sagault agonisant (p. 151) ou ceux de Marie Bliard (qui se remémore "les minuscules moments de bonheur », p. 189).

22. Par opposition aux personnages « reparaissants » de Balzac (voir par exemple Mireille LABOURET, «À propos des personnages reparaissants. Constitution du personnage et "sens de la mémoire" ", L'Année balzacienne, 6, 2005, p. 135-138). Une de ces (nombreuses) esquisses psychologiques se lit lors de l'évocation de la mort de Sagault (p. 148 et suiv.).

23. Jacques RANCIÈRE, Les mots de l'histoire. Essai de poétique du savoir, Paris, Éditions du Seuil, 1992, p. 163. Cette poétique absente réclamée par J. Rancière s'accomplit au reste dans 14 juillet en écho à une vision politique qui doit beaucoup aussi à celle du philosophe (cf. infra, notes 55 et 63). Rappelons que, dans ce passage, il est question du positionnement de l'histoire contemporaine face à celle de Jules Michelet, célébré, lui, pour son « travail littéraire antilittéraire " (Les mots de l'histoire..., p. 163). Toutefois, la poétique de Michelet n'est plus adaptée à notre époque, comme le remarque J. Rancière ( $«$ Michelet avait inventé une poétique pour une certaine historicité, pour la généalogie du sujet France et de la forme République », ibid., p. 166), ce qui peut expliquer que l'historien de la Révolution ne figure pas parmi les modèles d'É. Vuillard, loin s'en faut. Il s'agit dès lors pour ce dernier de « dessiner la figure de l'historicité propre à son âge » (ibid., p. 165).

24. Sur cette question, voir G. RUDÉ, La foule dans la Révolution française, op. cit., p. 74-77. Éric Vuillard y fait directement référence p. 182.

25. La problématique de la liste connaît depuis peu une certaine actualité dans les pratiques littéraires et dans les dynamiques de recherche. Ainsi cet objet a-t-il récemment été analysé dans diverses perspectives : philosophique (par ex. Bernard SÈvE, De haut en bas. Philosophie des listes, Paris, Éditions du Seuil, 2010), littéraire (Umberto ECO, Vertige de la liste, Paris, Flammarion, 2009), ou encore narratologique (voir entre autres Romain LORIOL, « Comment lire une liste ? Interroger les pratiques antiques du catalogue ", dans Romain Loriol et Marie Ledentu (éd.), Penser en listes dans les mondes grec et romain, Bordeaux, Ausonius, à paraître). Ces quelques exemples ne sont pas exhaustifs : voir pour plus de références le dossier " Aux listes et caetera ", Acta fabula, 14, 4, dossier critique $\mathrm{n}^{\circ} 28,2013$.

26. Tout du moins selon la forme que prend usuellement la liste. Comme le rappelle R. LORIOL ( Comment lire une liste ?...», art. cité), les listes présentent généralement des séquences discontinues, marquées par la parataxe ou l'asyndète $(\mathrm{X}, \mathrm{Y}, \mathrm{Z})$ ou, à l'inverse, par la polysyndète (et $\mathrm{X}$ et $\mathrm{Y}$ et $\mathrm{Z}$ ). Dans l'un ou l'autre cas, toutefois, il n'y a pas de relation de dépendance ou de hiérarchisation entre les éléments.

27. La synecdoque semble au contraire propre à l'individu bourgeois, qui porte sur lui les signes de son statut et n'est qu'extériorité : tel Louis-Lézin de Milly qui « est tout entier dans son petit mouchoir blanc» (p. 141). Le contraste avec le prolétaire Sagault quelques pages plus loin est 
évident: « son enveloppe est vide » (p. 148). L'impossibilité pour l'homme du peuple de faire signe explique son seul devenir possible dans le collectif de la foule.

28. « [Une ville] c'est une masse, une foule, cohue grisante, une flopée, une multitude », p. 77.

29. Pour qui le comportement de la foule est réglé par « la loi de l'unité mentale » de "l'âme collective » (G. LE BON, op. cit., p. 12 et 15).

30. Notamment en étudiant l'utilisation des anaphores dans les listes (anaphores en « oui, ici [...] » p. 15 ou en « il y avait » p. 87) ou celle des épiphores (en « [...] veut voir » p. 127), et en s'intéressant aux effets de rupture dans ces morceaux de bravoure. Comme le relève R. LORIOL ( Comment lire une liste ?...», art. cité), la liste se définit toujours par «l'homogénéité de [ses] items » et par «l'hétérogénéité formelle avec son environnement ».

31. Ainsi de Fournier et de Delorme (p. 69-71), ou de Claude Cholat et de Jean Rossignol : « ils sont à trois cents mètres l'un de l'autre. Ils ne se connaissent pas » (p. 119)

32. Sur ce point, voir Vincent RUBIO, « La Foule. Réflexions autour d'une abstraction », Conserveries mémorielles, 8,2010 , p. 5, qui rappelle que le paradoxe sorite (ou paradoxe du tas de sable) s'applique parfaitement aux problèmes de définition de la foule.

33. Cette liste s'effectue du point de vue bourgeois : " on compta le moindre bouton de porte disparu, chaque pelle à feu, chaque pincette, le plus petit morceau de tapisserie arraché, les nappes déchirées, les oreillers crevés [...]» (p. 19).

34. Pour utiliser le vocabulaire propre à la sociocritique : voir une introduction à cette discipline dans Régine ROBIN, «De la sociologie de la littérature à la sociologie de l'écriture : le projet sociocritique ", Littérature, 70, 2, 1988, p. 99-109.

35. "Ce doit être une foule prodigieuse, une sorte de totalité " (p. 72, nous soulignons), passage d'ailleurs immédiatement suivi d'une liste. Le but est donc bien de faire correspondre, à l'échelle micro-structurelle, le constat que " partout errait une foule bizarre » (p. 52), une foule qui se superpose à la ville, au peuple (p. 73 et p. 92), à « l'humanité » (p. 61 et p. 121).

36. « La foule court dans les jardins de la manufacture. On se presse entre les petits massifs vert tendre, on traverse la rivière de l'Inclination [...]» (p. 15) ; « mais la foule ne se calme pas. On est montés sur les toits, on a grimpé sur les réverbères [...]» (p. 97) ; « la foule dut reculer, se planquer même [...]. On dut se faire tout fifre derrière les arbres [...]. Oui, on dut courir, voler [...] » (p. 107) ; « la foule exaspérée mit alors le feu. [...] On crache ses poumons. On tire son tablier devant sa bouche » (p. 159) - relevé non exhaustif.

37. Olivier SOUTET, La Syntaxe du français, Paris, PUF, 2018 ( $7^{\mathrm{e}}$ éd.), p. 30.

38. On pourrait également ici dresser un parallèle avec des techniques stylistiques et linguistiques proches qui, dans d'autres langues, permettent de narrer la foule dans une perspective indéterminante: il en va ainsi de la récurrence du passif impersonnel chez les historiens et les poètes épiques latins quand on en vient aux masses agissantes.

39. Qui ménagent donc les deux pôles de cet axe uniformisation/individualisation en recourant simultanément à deux marqueurs syntaxiques contradictoires, le singulier du « on » et le pluriel de l'accord (par ex. " on avait été écartés des débats » page 12 ou " on est montés sur les toits » page 97).

40. Ainsi pour Fournier : « un type fait de grands gestes de ralliement » (p. 66) laisse la place à la page suivant à "ce type, c'est Fournier » (remarquer le démonstratif) ; idem pour Delorme, d'abord nommé « un type ", puis « un Noir », " le Nègre » et enfin « Delorme » : on voit ici à l'œuvre le mécanisme d'individuation, « l'un de ces élans généreux où l'homme [et non un homme] se substitue aux autres ». Même exemple pour Michel Béziers (p. 169) et, au reste, pour Camille Desmoulins (p. 45-46).

41. Éric VUILLARD, La Bataille d'Occident, Arles, Actes Sud, 2012, passim, en particulier page 102. La fin du chapitre « La foule » de 14 juillet semble, elle, dresser un prolongement avec les foules de l'ère industrielle via la mention des « zincs » et des « usines » (p. 93). 
42. Éric VUILLARD souligne page 76 de 14 juillet l'équivalence entre « l'expérience du grand nombre » et «l'anonymat ». Symboliquement, ce passage est directement suivi de l'expression d'une première personne du pluriel : «oui, désormais, nous sommes anonymes [...]».

43. Même phénomène page 64 : une foule muette et récalcitrante errait autour des murailles. On savait que la poudre était là [...]. Nous pourrons peut-être brûler du même feu, mais pas le même jour, pas la même heure [...]. »

44. Gérard GENETTE, Introduction à l'architexte, Paris, Seuil, 1979.

45. Paul RICœUR, Temps et Récit, I : L'intrigue et le récit historique, Paris, Seuil, 1991, p. 316 : « Raconter, c'est déjà expliquer. [...] Cette thèse de base a de nombreux corollaires. Si tout récit met en œuvre, en vertu même de l'opération de mise en intrigue, une connexion causale, cette construction est déjà une victoire sur la simple chronologie et rend possible la distinction entre histoire et chronique. En outre, si la construction de l'intrigue est œuvre de jugement, elle lie la narration à un narrateur, et donc permet au "point de vue" de ce dernier de se dissocier de la compréhension que les agents ou les personnages de l'histoire peuvent avoir eu de leur contribution à la progression de l'intrigue ; contrairement à l'objection classique, le récit n'est aucunement lié à la perspective confuse et bornée des agents et des témoins immédiats des événements ; au contraire, la mise à distance, constitutive du "point de vue", rend possible le passage du narrateur à l'historien. » La multiplication des modes de focalisations chez É. Vuillard générerait ainsi une confusion supplémentaire et féconde entre le récit historien et le récit esthétique.

46. On pense par exemple à la mise à distance du discours historiographique contrerévolutionnaire à l'égard de l'apparence des révolutionnaires, p. 57, sur laquelle nous revenons dans la partie suivante.

47. Ce plaidoyer pro domo du récit fait écho à certaines réflexions de Michel de Certeau, par exemple : «Tel est précisément le rôle premier du récit. Il ouvre un théâtre de légitimité à des actions effectives. Il crée un champ qui autorise des pratiques sociales risquées et contingentes. » (Michel DE CERTEAU, L'Invention du quotidien, 1. Arts de faire, Paris, Gallimard, 1988, p. 183)

48. Cet élargissement du temps du récit au temps de l'Histoire via les anachronismes sert avant tout une proposition politique tout actuelle d'É. Vuillard, sur laquelle nous revenons à la fin de cet article.

49. Dans un entretien avec Patrick Delorieux, É. Vuillard motive le recours à cette tonalité en l'articulant à une évolution historique et culturelle propre à ce moment : « Le lyrisme est l'une des expériences de la Révolution. La langue a changé. Elle doit être capable de convaincre, de donner à voir, d'emporter. Cela permet de sentir autrement, comme si les mots nous réveillaient. » («La Révolution selon Éric Vuillard», entretien avec Patrick Delorieux, 2016, disponible en ligne http://www.les-lettres-francaises.fr/2016/10/la-revolution-selon-eric-vuillard/, consulté le 07/01/2020)

50. Émile BENVENISTE, Problèmes de linguistique générale I, Paris, Gallimard, 1966, p. 241.

51. P. 130.

52. Dans un entretien avec Laurent Jeanpierre et Marielle Macé, É. Vuillard déclare: «Depuis longtemps, je désirais raconter un soulèvement. La littérature n'est pas faite pour ça : il lui faut un nombre réduit de personnages, une intrigue un peu linéaire et non pas un brouillard d'événements. C'est que l'art du récit n'a pas été inventé pour rapporter les mouvements de la foule mais pour célébrer les héros. " (Éric vUILLARD, " Nos infortunes sont coriaces ", entretien avec Laurent Jeanpierre et Marielle Macé, Critique, 838, 3, 2017, p. 215-226, en part. p. 225)

53. Nous renvoyons ici à tout un courant de critique littéraire qui s'intéresse par exemple aux « styles » de vie, à l'instar de Marielle MACÉ, Styles. Critiques de nos formes de vie, Paris, Gallimard, 2016, qui propose une entrée "Style et colère, style et histoire ». Il n'est sans doute pas anodin que la même chercheuse ait consacré récemment tout un texte à Éric Vuillard (Marielle MACÉ, «Le chant sinistre de la conquête », Critique, 838, 3, 2017, p. 202-214) : on donc peut imaginer une 
analyse de la foule comme "forme de vie ". C'est ce que fait par exemple le Comité invisible, notamment dans Maintenant, (Comité invisible, Maintenant, Paris, La fabrique, 2017). Dans le monde anglo-saxon, la critique se regroupe autour d'une " affect theory ", qui s'intéresse aux sentiments " personnels » (feelings), aux émotions " sociales », et aux affects " pré-personnels ». On citera ici seulement, parmi un corpus grandissant et pour ses études de textes littéraires, Martha NussBaum, Upheavals of Thought. The Intelligence of Emotions, Cambridge, Cambridge University Press, 2001.

54. Le roi, lui, dispose de " quatre horlogers ", dont "l'un d'eux a pour unique mission, chaque matin, de remonter sa montre » (p. 35). Et Vuillard d'ajouter ce commentaire ironique tout autant que colérique : « On dirait une farce, une rabelaiserie, absurdité d'auteur, un racontar. » 55. On retrouve ici la donnée même du politique, selon Jacques Rancière : « Le dissensus, c'est la reconfiguration des rapports entre sens et sens, c'est-à-dire entre présence sensible et signification. Il peut se décrire comme un excès, un mécompte par rapport au compte ordonné des corps et des significations. En politique, ce mécompte prend la forme d'introduction de sujets qui n'étaient pas comptés et qui, en se donnant un nom, se font compter comme aptes à compter les choses et les êtres qui font partie de la communauté, à re-découper le donné des situations, à changer les noms qu'on peut leur donner. » (Jacques RANCIÈRE, Entretien autour de Politique de la littérature avec Lionel Ruffel, Vox Poetica, 2007, disponible en ligne http://www.vox-poetica.org/ entretiens/intRanciere.html, consulté le 07/01/2020)

56. Les passages mentionnés se trouvent page 57 de 14 juillet et reproduisent l'extrait de Galort de Montjoie que l'on trouve dans Hippolyte TAINE, Les Origines de la France contemporaine. L'ancien régime. La Révolution : L'Anarchie - La conquête jacobine, Paris, Robert Laffont, 1986 [1 $1^{\text {re }}$ édition 1881], p. 331 (livre premier, chapitre II, I). Sont ainsi évoqués un « nombre effrayant d'hommes mal vêtus et de figure sinistre " et une "quantité d'étrangers venus de tous les pays, la plupart déguenillés, armés de grands bâtons ».

57. Nous n'exagérons pas, il suffit de citer Jules MICHELET, Histoire de la Révolution française, I, Paris, Gallimard, 1952, p. 151 : «L'homme, c'était Thuriot, un dogue terrible, de la race de Danton; nous le retrouverons deux fois, au commencement et à la fin ; sa parole est deux fois mortelle : il tue la Bastille, il tue Robespierre. "

58. L'épisode de la chemise arrachée n'est pas sans faire écho, peut-être, à un événement contemporain analogue, lors des manifestations des employés d'Air France, le 5 octobre 2015. Ce ne serait guère surprenant de la part d'Éric Vuillard, dont l'écriture s'opère souvent comme une réaction épidermique à l'actualité : témoin la publication anticipée de La Guerre des pauvres en janvier 2019 (Arles, Actes Sud) due à une volonté de synchronisation avec le mouvement des gilets jaunes (cf. un entretien au Monde datant du 19 janvier 2019, disponible en ligne https:// www.lemonde.fr/culture/article/2019/01/19/eric-vuillard-ecrire-pour-faire-chavirer-lemonde_5411525_3246.html, consulté le 07/01/2020).

59. À moins qu'on ne l'identifie ainsi (p. 198) : «Les témoignages du jour parlent d'une ambiance folle, exubérante, jamais vue. La joie. » C'est elle qui se retrouve dans l'appel à jeter les papiers par les fenêtres des dernières pages (p. 200, nous soulignons) : "Ce serait beau et drôle et réjouissant. Nous les regarderions tomber, heureux, et se défaire. »

60. Voir p. 16 , p. 37 , p. $54-55$, p. $59-60$, p. 64 , p. 92 , p. 103 , p. 119 , p. 125 , p. 127 , p. 163 , et p. $199-200$.

61. Voir ainsi par exemple : «Pour Spinoza la multitudo désigne une pluralité qui persiste comme telle sur la scène publique, dans l'action collective, dans la prise en charge des affaires communes, sans converger vers un Un, sans s'évaporer sur un mode centripète. Multitude est la forme d'existence sociale et politique du Nombre en tant que Nombre: forme permanente, non épisodique ou interstitielle. Pour Spinoza, la multitudo est la clef de voûte des libertés civiles. » (Paolo VIRno, Grammaire de la multitude. Pour une analyse des formes de vie contemporaines, Paris, François Maspero, 2002, p. 8) 
62. Ce dernier aussi est lié à la pensée spinoziste, dont on sait par ailleurs le rôle primordial qu'elle joue dans la réflexion contestataire : de Louis Althusser à Frédéric Lordon en passant par Toni Negri. Voir par exemple : «S'il existe une rationalité de la politique, elle ne peut consister qu'en tant que prise en compte de la conflictualité irréductible des passions humaines telles qu'elles se manifestent dans le désir de la multitude ou du "peuple" de ne pas être opprimé par le souverain. " (Saverio ANSALDI, «Conflit, démocratie et multitude : l'enjeu Spinoza Machiavel », Multitudes, 27, 4, 2006, p. 219, nous soulignons)

63. Dans un livre brillant, Andrew Gibson a fait l'analyse de ce concept de la raison historique, différent de celui de progrès (Andrew GIBSON, Intermittency. The Concept of Historical Reason in Recent French Philosophy, Édimbourg, Edinburgh University Press, 2012). On trouve ainsi chez É. Vuillard une vision quasi ranciérienne de la politique comme processus d'émancipation, où le soulèvement joue un rôle central (voir Jacques RANCIÈRE, Aux bords du politique, Paris, Gallimard, 2004). De fait, d'intéressants points d'accroche émergent à la lecture entre 14 juillet et un bref essai du philosophe, dans le catalogue de l'exposition Soulèvements dirigé par Georges DidiHuberman, où J. Rancière s'interroge : "Qu'y a-t-il au monde qui ne se soulève ? " Jacques RANCIÈRE, «Un soulèvement peut en cacher un autre », dans Georges Didi-Huberman, Catalogue de l'exposition Soulèvements, Paris, Gallimard/Jeu de Paume, 2004, p. 63-70, en part. p. 63) Comme chez É. Vuillard avec l'image de la pluie de papier à la fin du récit, le soulèvement n'est pas que populaire : il faut voir « le chemin qui va du tournoiement des feuilles à la tourmente des âmes et des tempêtes sous les crânes à l'insurrection dans les rues " (ibid.). "Tourmente des âmes ", « tempêtes sous les crânes $»:$ J. Rancière développe dans cette contribution une pensée du pathos comme praxis en puissance (ibid. : p. 64) qui, encore une fois, fait lien avec l'image de la foule dans 14 juillet.

64. En langue, le Grand Robert nous dit 1876. En histoire, Christian Topalov n'est pas éloigné (cf. Christian topalov, Naissance du chômeur : 1880-1910, Paris, Albin Michel, 1994).

65. Voir le site des éditions Actes Sud: https://www.actes-sud.fr/catalogue/litterature/14-juillet (consulté le 07/01/2020).

\section{RÉSUMÉS}

Cet article se propose d'analyser 14 juillet d'Éric Vuillard à la lumière de la réception et de la représentation originale de la prise de la Bastille qu'il propose. Dans ce « récit », qui n'est pas un roman, l'auteur s'engage dans un rapport polémique à l'historiographie aussi bien contrerévolutionnaire que républicaine de la Révolution française, et défend l'idée que la littérature peut rendre compte de façon plus juste des réalités du soulèvement du 14 juillet. Nous concentrant sur la figure de la foule, qui est au cœur du récit, nous proposons d'étudier l'effort d'É. Vuillard pour aboutir à une poétique nouvelle de l'action collective. Après avoir rappelé les modèles littéraires que l'auteur met à distance, nous rendons compte des propositions stylistiques novatrices de l'œuvre, avant d'en venir à l'instabilité générique significative qui s'y joue. Enfin, nous mettons au jour l'enchevêtrement de multiples discours sur la foule révolutionnaire, qui aboutit à une défense de l'universalité du soulèvement.

This paper aims to analyse the reception of the Storming of the Bastille in Éric Vuillard's 14 juillet. In this work defined as a "narrative" (and not as a novel), the author raises a debate over the conservative as well as liberal 19th-century historiography of the French Revolution, 
and promotes the idea that literature should be recognised the ability of depicting more accurately the revolutionary insurrection. We therefore focus on the specific poetic of the crowd, which the author forges as a rehabilitation of collective action. In the first part of this paper, we put back this original stylistic proposal within the broader framework of the representation of the crowd; then we examine the unstable genre peculiar to a work whose main character is the crowd. Finally, we take apart the many layers of discourse on the revolutionary mob, shifting the focus to the auctorial discourse that postulates the universality of the insurrection.

\section{INDEX}

Keywords : crowd, historiography, poetics, insurrection, Bastille

Mots-clés : foule, historiographie, poétique, insurrection, 14 juillet

\section{AUTEURS}

\section{LOUIS AUTIN}

Université Grenoble Alpes, Universität Osnabrück

Litt\&Arts - Translatio, UMR 5316

\section{PIERRE-VICTOR HAURENS}

ENS Lyon

CERCC, EA 1633

YVES SCHULZE

ENS Lyon - Université de Lyon-III

CERCC, EA 1633 